\title{
AVALIAÇÃO DO RISCO TOXICOLÓGICO ATRAVÉS DA MODELAGEM DINÂMICA DA EXPOSIÇÃO AOS COMPOSTOS BTEX
}

\author{
TOXICOLOGICAL RISK DETERMINED BY DYNAMIC MODELING OF EXPOSURE TO BTEX \\ COMPOUNDS
}

\author{
Ademir da Silva Santos ${ }^{1}\left(^{*}\right)$ \\ Fermin Garcia Velasco ${ }^{2}$ \\ Francisco Heriberto Martínez Luzardo ${ }^{3}$ \\ Sérgio Fred Ribeiro Andrade 4 \\ Luis Nieto González ${ }^{5}$
}

\section{Resumo}

A mensuração do risco toxicológico é um método científico que utiliza processos empíricos, experimentais e modelagens computacionais para aferição dos efeitos adversos de determinadas substâncias à saúde humana. Estes efeitos podem ser de natureza carcinogênica e não carcinogênica. Os compostos BTEX (benzeno, tolueno, etilbenzeno e xilenos), presentes no diesel e gasolina, possuem extrema relevância ambiental devido ao impacto toxicológico que podem provocar. $\mathrm{Na}$ decorrência de derramamentos ou vazamentos acidentais de combustíveis, esses compostos entram em contato com o solo e/ou água, movendo-se rapidamente até atingir as zonas de exposição humana, que por definição sintética se dão pelo contato com o solo, água e ar. Os métodos utilizados para aferição dos níveis de risco em cada via de exposição são usualmente estáticos quanto ao tempo e níveis de concentração. A metodologia aplicada neste trabalho é uma adaptação do modelo ACBR da CETESB, juntamente com metodologias de modelagem ambiental e computacional da U.S. EPA. O resultado apresentado produziu uma metodologia dinâmica de análise do risco toxicológico, quanto aos níveis de concentração e tempo

1 Doutorado interrompido; Desenvolvimento e Meio Ambiente; Universidade Estadual de Santa Cruz, UESC, Brasil; Estudante da Universidade Estadual de Santa Cruz, UESC, Brasil; Endereço: Instituto Federal da Bahia, Departamento de Administração do IFBA, Campus Porto Seguro. Br 367, KM 57,5, Fontana 145810000 - Porto Seguro, BA - Brasil E-mail: adm_santos@yahoo.com.br $\quad(*)$ Autor para correspondências

2 Dr.; Ciencias Físicas; Centro de Aplicaciones Tecnológicas y Desarrollo Nuclear, CEADEN, Cuba; Professor Pleno da Universidade Estadual de Santa Cruz, UESC, Brasil; Universidade Estadual de Santa Cruz, Departamento de Ciencias Exatas e Tecnologicas; Endereço: Rodovia Ilheus-Itabuna Km 16, UESC, Torre Central, $6^{\circ}$ Andar, FIMED. Salobrinho, 45662000 - Ilhéus, BA - Brasil E-mail: fermingv@gmail.com

3 Dr.; Ciências Técnicas; Centro Nacional de Pesquisas Científicas, CNIC, Cuba; Professor titular na Universidade Estadual de Santa Cruz, UESC, Brasil; Endereço: Universidade Estadual de Santa Cruz, DCET. Rodovia Ilheus-Itabuna Km 16, Salobrinho 45662000 - Ilhéus, BA - Brasil; E-mail: fmartinezluzardo@gmail.com

4 Dr.; Ciências Ambientais; Universidade Estadual de Santa Cruz, UESC, Brasil; professor adjunto do Departamento de Ciências Exatas e Tecnológicas da UESC, tem experiência na área de computação, em análise e sistemas de informações, banco de dados corporativo, data warehousing, data mining, engenharia de softwaree geoinformática comaplicação de modelos multivariados, redes neurais e máquinas de vetores; Endereço: Universidade Estadual de Santa Cruz, DCET. Rod. Ilhéus-Itabuna, km 16, Salobrinho 45662000 - Ilheus, BA - Brasil E-mail:sergio.fred@hotmail.com

5 Dr.; Física; Universidade Estadual de Campinas, UNICAMP, Brasil; Professor Adjunto da Universidade Estadual do Sudoeste da Bahia, UESB, Brasil; endereço: Universidade Estadual do Sudoeste da Bahia, Departamento de Estudos Básicos e Instrumentais - DEBI - Itapetinga. UESB - Itapetinga, Campus Itapetinga 45700000 - Itapetinga, BA - Brasil E-mail: Inietog@gmail.com

\begin{tabular}{llllll}
\hline Ambiência & Guarapuava (PR) & v.14n.1 & p.154-173 & Jan/Abr 2018 & ISSN $1808-0251$
\end{tabular}


de exposição, inserindo parâmetros de ajustes que evidenciam as elevações temporais do risco potencial para cada composto BTEX.

Palavras-Chave: risco toxicológico, BTEX, Hydrus 1D, areia silicosa.

\section{Abstract}

The measurement of the toxicological risk is a scientific technique that uses empirical methods, experimental and computational modeling to measure the adverse effects of substances on human health. These effects may be at the nature carcinogenic and not carcinogenic. Among the elements with greater toxicological power, the BTEX compounds (benzene, toluene, ethylbenzene and xylenes), have extreme environmental importance, since they are present in diesel and gasoline fuels. In result of spills or accidental fuel leaks, these compounds come into contact with soil and / or water moving quickly to reach areas of human exposure, which in short definition occur by contact with soil, water and air. The methods used to measure risk levels in each route of exposure are usually static in terms of time and concentration levels. The methodology applied in this work is an adaptation of CETESB's ACBR model, together with environmental and computational modeling methodologies of U.S. EPA. The result presented produced a dynamic methodology of toxicological risk analysis, regarding concentration levels and time of exposure, inserting parameters of adjustments that evidence the temporal rises of the potential risk for each BTEX compound.

Key words: toxicological risk, BTEX, Hydrus 1D, silica sand

\section{Introdução}

Os processos de avaliação do risco toxicológico à saúde humana são ferramentas preditivas de baixo custo, fundamentais para gestão ambiental. Normativamente, devem ser aplicadas de forma obrigatória nos procedimentos de avaliação do impacto ambiental. Possuem como pressupostos básicos: observações em laboratório e em campo, métodos de informação e extrapolação de doses em animais e realização de estimativas de exposição e caracterização de populações receptoras (PORTO e FREITAS apud CANTER, 1997).

O risco toxicológico é um conceito probabilístico que define o potencial de ocorrência de efeitos carcinogênicos e não carcinogênicos. Esses efeitos são dependentes de uma relação entre perigo e exposição (MIHELCIC e ZIMMERMAN, 2012). Nos processos de estimação do risco toxicológico à exposição a determinados contaminantes é dependente de aspectos investigatórios prévios, como, os cenários específicos de contaminação, as vias de exposição, as características dos receptores (peso e idade), duração e frequência (magnitude e tempo) e mecanismos de absorção (inalatória, dérmica e oral) (PNUMA/IPCS, 1999).

Alguns contaminantes, como os compostos BTEX (benzeno, tolueno, etilbenzeno e xilenos) existentes nos combustíveis, diesel e gasolina, elevam a complexidade de estimação da exposição e do risco toxicológico, devido às propriedades de reação no solo, como a solubilidade, a volatilidade, a densidade e os coeficientes de partição octanol/água e octanol/carbono (U.S. EPA, 1996; CETESB, 2001; KAIPPER, 2003). O conjunto de contaminantes BTEX é denominado como compostos orgânicos voláteis (COV) ou hidrocarbonetos aromáticos voláteis (HAV), classificados 
como carcinogênicos (benzeno e etilbenzeno) e não carcinogênicos (tolueno e xilenos). Quando infiltrados no solo criam uma pluma de contaminação que flutua acima do nível freático, sofrendo processos de degradação e atenuação que transportam frações do contaminante para outras regiões do solo e até mesmo para outros compartimentos como, o ar no ambiente externo ao solo, na água subterrânea e nas partículas do solo de forma residual (DONAIRE, 2007; PINHO, 2009; PENNER, 2000; FINOTTI et al, 2001).

A metodologia de avaliação do risco toxicológico da U.S. EPA (1989), tem sido adotada em muitos países, inclusive no Brasil (CETESB, 2001). Em particular, para contaminação por hidrocarbonetos de petróleo e outros combustíveis líquidos, foi desenvolvido o método RBCA Risk Based Corrective Action at Chemical Release Sites (ASTM, 2010), adotado no Brasil (CETESB, 2006). Esta abordagem introduz o conceito de cenários de exposição baseados em modelos de transporte de contaminantes no meio ambiente, que possibilita uma análise flexível do processo de infiltração do contaminante. Essa metodologia possui um enfoque conservador, que considera a concentração máxima como um valor fixo no tempo e que fornece uma estimativa segura para o risco, mas, que pode ser sobrestimada. Neste enfoque, a dependência temporal das concentrações dos contaminantes é ignorada. A estimação incorreta (subestimado ou sobrestimado) do risco faz da sua gestão, um processo mais complexo e dispendioso. Por outro lado, metodologias como da CETESB (2006) fixam os tempos de exposição com base apenas nos dias úteis de trabalho em cenários urbanos e rurais, que variam de 250 a 350 dias. Esse entendimento não reflete a realidade do processo de infiltração e decaimento das concentrações dos contaminantes, por exemplo, dos compostos BTEX, que são dependentes do tempo.

Dentro do processo de avaliação de risco, um dos elementos fundamentais se refere à modelagem do transporte do contaminante entre os compartimentos do solo (Fases sólida, líquida e vapor). Para isso diversos modelos computacionais são utilizados (SESOIL, Modflow, Hydrus 1D, HST3D, entre outros), cujo objetivo é estimar o transporte e concentrações de solutos em cada estado ambiental, a partir do qual é avaliada a exposição e a quantificação do risco. Em especial, o Hydrus 1D (ŠIMU゚NEK et al, 2008), foi utilizado em vários trabalhos com diversos tipos de solutos, como fertilizantes, pesticidas, metais e outros compostos orgânicos, em diferentes matrizes de solo (ŠIMŮNEK e BRADFORD, 2008; MALLANTS et al, 2011; LEIJ et al, 2011; ZON, 2012; RUBIO, 2005; CHAVES, 2009; MINELLA, 2010).

O presente trabalho quantificou e analisou o risco toxicológico da exposição ao BTEX, considerando a dinâmica de alteração temporal das concentrações nos compartimentos ambientais; incluído um estudo do transporte dos contaminantes no ar, solo residual e água subterrânea. Foi aplicado o Hydrus 1D (ŠIMŮNEK et al, 2008) em conjunto com a metodologia ACBR (U.S. EPA, 1989). Pretendeu-se, portanto, estimar as probabilidades de risco toxicológico com efeitos carcinogênicos e não carcinogênicos ocasionados pela exposição a esses compostos.

\section{Material e métodos}

O estudo foi aplicado na área experimental da jazida de areia silicosa identificada no município de Belmonte, Estado da Bahia, Brasil (MOREIRA, 1997). A metodologia foi organizada com base nas etapas do processo de avaliação do risco toxicológico. Inicialmente, foram realizados ensaios experimentais para obtenção dos parâmetros físicos e hidráulicos do solo, utilizados pelo modelo Hydrus 1D conforme Van Genuchten (1980). Este modelo utiliza o teor de umidade residual e saturada e a condutividade hidráulica saturada como parâmetros de influência direta no processo de infiltração de solutos no solo. Algumas propriedades dos contaminantes, 
como o coeficiente de Henry e o coeficiente de adsorção, foram definidos com base no banco de dados IRIS - Integrated Risk Information System, da U.S. EPA (2002). Foi considerada, também, a influência dos fatores atmosféricos. A precipitação, evapotranspiração e a temperatura, foram obtidas do INMET (2012), no período compreendido entre o início de outubro 2012 e final de setembro de 2013.

Os parâmetros demográficos e sócios - econômicos dos modelos da avaliação da exposição como: peso, idade, duração e frequência de exposição, tempo máximo de exposição, fatores de absorção dérmicos, inalatórios e orais, foram adotados dos padrões reportados pela CETESB (2001). A caracterização do risco toxicológico foi realizada, considerando todas as vias de exposição e absorção dos compostos BTEX.

\section{Modelo de transporte hidráulico}

O Hydrus 1D é um modelo computacional unidimensional, aplicado em solos de porosidade média, em particular os arenosos (SUÁREZ et. al, 2007; ŠIMŮNEK et al, 2008; SAIFADEEN e GLADNYEVA, 2012). O modelo hidráulico considera os processos de advecção e dispersão, de fluxo e divisão de massas entre fases, descritos pela equação de controle geral do transporte de solutos (VAN GENUTCHEN, 1980, JURY et al, 1983). O Hydrus 1D também inclui no modelo de transporte de solutos, os parâmetros de fluxo variável da equação de Darcy-Buckingham, conforme Van Genuctchen (1980), sendo um modelo computacional aplicável em diferentes texturas de solo e para diversos tipos de solutos.

O modelo de transporte de solutos exige o conhecimento prévio das características de retenção e condutividade hidráulica saturada do solo, inclui reações de difusão em água e gás, com processos de decaimento de primeira ordem, onde a velocidade de infiltração depende da concentração do soluto e do fluxo de água, que no modelo Hydrus 1D é vertical (ŠIMŮNEK et al,2008; PIGGOTT e CAWLFIELD, 1996). A curva de retenção (variação do teor de umidade) e a condutividade hidráulica são estimadas conforme Van Genuchten - Mualem (1980), em meio saturado e não saturado.

O transporte geral de solutos e as concentrações relativas a cada fase do solo (sólida, gasosa e líquida), são quantificados pelo Hydrus 1D (JURY et al,1983; JURY et al, 2004; TODD e MAYS, 2005). Os fatores como precipitação, evapotranspiração, temperatura, também são considerados pelo modelo como parâmetros que influenciam na velocidade potencial do fluxo de água no solo.

\section{Caracterização experimental do solo}

O plano de amostragem foi realizado em jazidas de areia silicosa inseridas na zona rural do município de Belmonte - BA, Brasil, conforme a metodologia SRS - Stratified Random Sampling da U.S.EPA (1989), em duas profundidades distintas (0 a $30 \mathrm{~cm}$ e de 30 a $640 \mathrm{~cm}$ ), considerando apenas dois horizontes estratigráficos: zona A e B do solo de estudo (MOREIRA, 1997).

As propriedades físicas e hidráulicas do solo (Tabela 1) foram obtidas a partir de ensaios laboratoriais: teor de umidade, textura granulométrica, sedimentação e densidade de campo, pelo método da EMBRAPA (1997). A condutividade hidráulica saturada foi realizada pelo método de Klute e Dirksen (1986). 
Tabela 1 - Parâmetros físicos das amostras de solo.

\begin{tabular}{ccccccc}
\hline $\mathbf{H}$ & $\begin{array}{c}\text { Nivel } \\
\mathbf{( c m )}\end{array}$ & $\begin{array}{c}\text { Textura } \\
\mathbf{( \% )}\end{array}$ & $\begin{array}{c}\mathbf{B D}(\mathbf{g} / \\
\left.\mathbf{c m}^{3}\right)\end{array}$ & $\mathbf{Q r}$ & $\mathbf{Q s}$ & $\mathbf{K}_{\mathbf{s}}(\mathbf{c m} / \mathbf{d i a s})$ \\
\hline A & $0-30$ & $\begin{array}{c}89,25 \text { areia } \\
10,75 \text { silte }\end{array}$ & 1,52 & $0,0025-0,0419$ & $0,3517-0,5292$ & $360,29-549,75$ \\
\hline \multirow{3}{*}{ B } & $30-640$ & $\begin{array}{c}86,00 \text { areia } \\
13 \text { silte } \\
1 \text { argila }\end{array}$ & 1,52 & $0,0024-0,0402$ & $0,3750-0,5226$ & $360,29-461,40$ \\
\hline
\end{tabular}

Nota:Hsãoos horizontes/faixas do solo divididosem doisníveis de profundidade,BDéa densidadedecampo, Qréoteordeumidade residual,Qséoteordeumidade saturada, $\mathrm{K}_{\mathrm{s}}$ éa condutividadehidráulica saturada eSSCécomposiçãotextural com silte, areiaeargila em inglês.

\section{Cenários dinâmicos e concentrações de exposição.}

Foram considerados os fatores de atenuação: fator de volatilização do solo superficial, subsuperficial e da água subterrânea para o ambiente externo e o fator de lixiviação do solo subsuperficial para água subterrânea, de acordo com a metodologia RBCA (ASTM, 2010; CETESB, 2006). Os parâmetros de atenuação para o BTEX provêm do banco de dados IRIS U.S. EPA (2004).

Os cenários de exposição foram modelados conceitualmente, com base nos cenários de exposição, considerando a fonte de contaminação, a partir do solo superficial, subsuperficial e água subterrânea, com aplicação de um ou mais fatores de atenuação natural (NAF) (CETESB, 2006).

\section{Estimativa do risco toxicológico.}

O método geral de estimação das doses de ingresso, que são as frações de contaminantes ingeridas, inaladas ou absorvidas (vias de introdução) para cada indivíduo exposto, durante um período de tempo, considera as concentrações de exposição em seus valores máximos fixos no tempo (ASTM, 2010, CETESB, 2001, PNUMA, 1999, WHO, 2000). Entretanto, em casos de acidentes ambientais, com exposições agudas, as concentrações dos contaminantes, nos diferentes compartimentos ambientais, sofrem variações temporais significativas, que não são consideradas no método geral de estimação das doses de ingresso (1).

$$
I=C \times \frac{I R \times E F \times E D}{B W \times A T}
$$

Onde, C, é a concentração de exposição (crítica ou média para um período de tempo determinado); IR, é a taxa diária de introdução por inalação; ingestão ou contato de água, ar e solo, EF, é a frequência de exposição estimada em dias úteis por ano; ED, é a duração de exposição baseada no tempo de atividade total de trabalho (anos); BW, é o peso corporal médio dos possíveis receptores e AT é o tempo médio total de vida dos receptores (estimado em dias).

No presente trabalho, se propõe uma alteração na metodologia, que considere a dependência temporal das concentrações de contaminantes no meio ambiente durante uma exposição acidental Consideraremos as doses de ingresso, neste cenário, como um fator dinâmico dependente do tempo 
$\left.\left.I_{d}(t)\right) I_{d}(t)\right)$. Como consequência, a expressão (2) passa a considerar dois processos de exposição, um relacionado a uma dose de ingresso máxima ou média fixa $(I)$ e outra relacionada com a estimativa dinâmica das doses de ingresso, através da variação temporal da concentração de contaminantes (3) num cenário de exposição acidental.

$$
I_{d}(t)=\left[\int_{t_{i}}^{t_{f}} c(t) d t\right] \frac{I R \times E F \times E D}{B W \times A T}
$$

A dependência temporal (termo entre colchetes) da concentração $C(t) C(t)$ num acidente pode ser ajustada por uma exponencial decrescente que contenha um parâmetro vinculante ao contaminante específico (d), equação (3), onde $C_{0} C_{0}$ é a concentração inicial do contaminante no momento do acidente.

$$
C(t)=C_{n} e^{-b t}
$$

A nova abordagem metodológica permite ter em conta a variação da concentração do contaminante em relação à frequência de exposição $(\mathrm{EF})$, o que possibilita a avaliação dinâmica da exposição e direciona a caracterização do risco a partir da evolução ou decaimento real das concentrações de contaminantes em casos de acidentes ambientais.

Quando o cálculo das doses de ingresso é efetuado, o valor refere-se à média acumulada para uma determinada concentração de exposição em um tempo proporcional (frequência de exposição específica), conforme equação (1), considerando uma exposição derivada de concentrações constantes e fixas. A frequência de exposição (EF) é contada como um valor representativo médio do nível de concentração. $\mathrm{O}$ modelo ora proposto, denominado risco dinâmico proporcional, preconiza a possibilidade de uma exposição dinâmica a níveis de concentração que podem sofrer picos de exposição, isto é, a partir das concentrações ora fixadas pelo método padrão, considera-se as elevações em seu limiar por meio de possíveis acidentes ambientais.

Em situações de derrames acidentais de altas concentrações de BTEX no solo, haverá uma rápida infiltração com processos de atenuação vinculados. $\mathrm{O}$ risco dinâmico proporcional consegue medir de forma gradual o risco toxicológico para os compostos, sendo possível estimar doses de ingresso em tempos curtos de exposição.

A caracterização do risco toxicológico é realizada a partir dos efeitos adversos prováveis a saúde humana, que podem ser carcinogênicos e não carcinogênicos. Os compostos, benzeno e etilbenzeno, são prováveis carcinogênicos humanos e o tolueno e xilenos são prováveis não carcinogênicos humanos, grupos 1 e 3 respectivamente, conforme IARC (1992). A metodologia ACBR da CETESB (2006) utiliza fatores de declividade carcinogênicos, slope factor (SF) como produtos aplicados sobre as doses de ingresso de compostos carcinogênicos (C), e para os compostos não carcinogênicos (NC), aplicam-se as doses de referência (RfD) indexadas como limite mínimo (oral, dérmica e inalatória), onde não ocorrem efeitos adversos à saúde humana (tabela 2).

\section{Tabela 2 - Fatores carcinogênicos (C) e doses de referência (NC) para os compostos BTEX.}




\begin{tabular}{cccc}
\hline Não Carcinogênicos & $\mathbf{R} f \mathbf{D}_{\text {oral }}$ & $\mathbf{R} f \mathbf{D}_{\text {dermal }}$ & ${\mathbf{R} f \mathbf{D}_{\text {inalatória }}}^{\text {Tolueno }}$ \\
Xilenos & 0,2 & 0,16 & 0,114 \\
Carcinogênicos & 0,2 & 0,184 & 0,0286 \\
Benzeno & $\mathrm{SF}_{\text {oral }}$ & $\mathrm{SF}_{\text {dermal }}$ & $\mathrm{SF}_{\text {inalatória }}$ \\
Etilbenzeno & 0,055 & 0,0567 & 0,0273 \\
\end{tabular}

Fonte: Banco de dados IRIS U.S. EPA (2004).

A caracterização da avaliação do risco é realizada com a aplicação das equações (6) e (7), sempre estimando o risco toxicológico para cada rota de exposição adotada nos cenários modelados, como também considerando o risco total para todas as vias de exposição.

A equação (4) é utilizada no cálculo do quociente de risco (HQ), sendo necessário o conhecimento das doses de referência $\left(R f D_{i}\right)$ para cada composto $B T E X$, considerando as vias, oral, dermal e inalatória (Tabela 2), como também das doses de ingresso já calculadas previamente conforme equações dinâmicas (2 e 3) (CETESB, 2001; BRILHANTE e CALDAS, 1999).

$$
H Q=\frac{I_{n}}{R f D_{i}}
$$

Para o risco toxicológico com efeitos adversos carcinogênicos a saúde humana é necessária à aplicação de fatores carcinogênicos $(\mathrm{C})$, denominados de slope factor $(S F)$. O $S F$ é uma probabilidade de resposta toxicológica, geralmente medida com base em um peso da evidência de câncer (mg/kg.dia) (BRILHANTE e CALDAS, 1999). Desta forma, o risco toxicológico foi quantificado através da equação (5):

$$
\text { Risco }=I_{n} \times S F
$$

Ao final as probabilidades de risco individuais, assim como os índices de perigo por via de exposição foram somadas, conforme aplicação das equações (6) e (7), onde é realizada a soma dos riscos individuais por via de exposição e a soma dos quocientes de risco por via de exposição.

Obtêm-se o risco total $\left(\right.$ Risco $\left._{t}\right)$ considerando todas as vias de exposição para compostos classificados como cancerígenos e o índice de perigo total $(H)$ considerando todas as vias de exposição para os compostos classificados como não cancerígenos humanos.

$$
\begin{aligned}
& \text { Risco }_{t}=\sum \text { Risco }_{i} \\
& \boldsymbol{H}=\sum \frac{I_{n}}{\text { RfD }_{i}}
\end{aligned}
$$




\section{Resultados e discussões}

\section{Avaliação da concentração inicial por profundidade do solo.}

Os resultados apresentados mostram a avaliação do risco toxicológico dinâmico proporcional para os compostos BTEX, a partir de um cenário de exposição decorrente de um acidente pontual com disposição de 1000 litros de diesel e gasolina em um solo arenoso (areia silicosa), com profundidade máxima de $700 \mathrm{~cm}$, em um ambiente de exposição populacional rural (MOREIRA, 2005).

A simulação com o Hydrus 1D considerou um período de 365 dias, sendo que o modelo calcula as concentrações para valores anteriores a $24 \mathrm{~h}$. Conforme estudos de Mazzuco apud Kaipper (2004) as proporções de BTEX em 10001 de diesel são: 9,79 mg benzeno, 21,02 mg tolueno, 6,90 mg etilbenzeno e 9,37 mg xilenos. Para 10001 de gasolina as proporções são: 13,88 $\mathrm{mg}$ benzeno, 22,47 mg tolueno, 2,58 $\mathrm{mg}$ etilbenzeno e 1,50 mg xilenos. O modelo apresentado também pode calcular os níveis de concentração em derrames de menor escala e/ou em vazamentos como os que ocorrem em tanques reservatórios de postos de combustível. No entanto, o cenário de exposição de risco aplicado neste trabalho, configura-se em um acidente ambiental com derrame expressivo de combustível. O comportamento das infiltrações dos compostos BTEX nas faixas $(30,150$ e $640 \mathrm{~cm})$ do solo utilizando as proporções no diesel segue representado na figura (1), sendo S1 (benzeno), S2 (tolueno), S3 (etilbenzeno) e S4 (xilenos).

\section{Figura 1 - Infiltração de BTEX nas profundidades 30, 150 e $640 \mathrm{~cm}$ proporcionais S1 (benzeno), S2 (tolueno), S3 (etilbenzeno) e S4 (xilenos).}
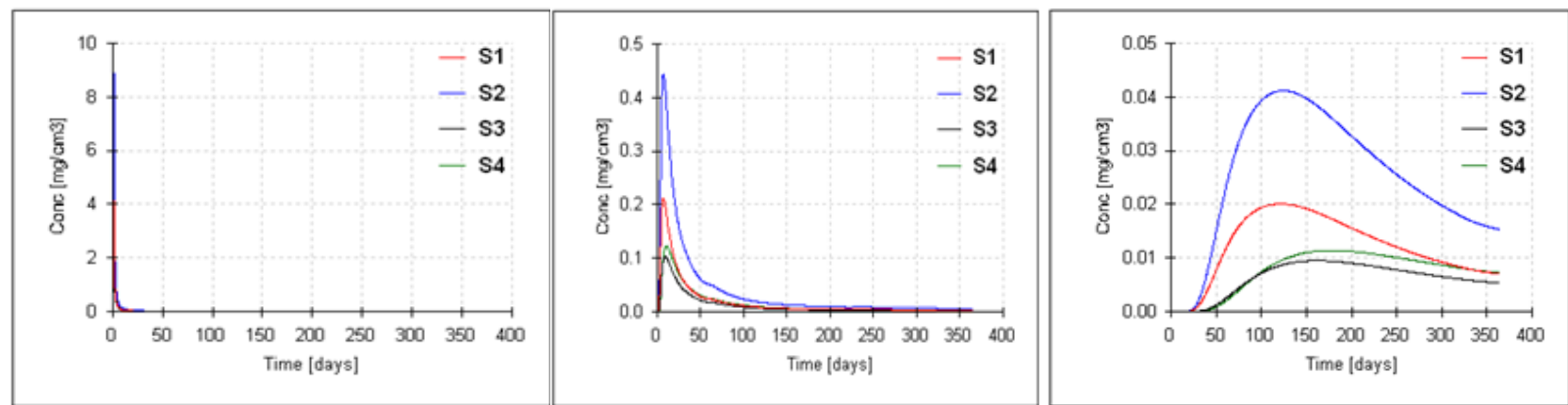

De forma distinta da metodologia geral tradicional a metodologia proposta de caracterização do risco toxicológico, presente nos resultados, contempla uma concentração de exposição dependente do tempo, ou seja, o risco toxicológico evolui conforme o tempo e acumulo médio das concentrações de exposição. Salienta-se que no caso de um acidente haverá uma concentração inicial elevada, com posterior decaimento gradativo da mesma (MAZZUCO, 2004; PIGGOTT E CAWLFIELD, 1996).

No caso dos resultados mostrados na figura (1) abaixo, a dependência temporal da concentração $C(t) C(t)$ se ajusta perfeitamente pela função proposta na equação (3), onde b é um parâmetro dependente do elemento contaminante. Ou seja, o valor de b é diferente para o benzeno e tolueno, por exemplo. Na figura (2) mostramos os resultados, como exemplos, destes ajustes para dois dos compostos BTEX para profundidades de 30 e $150 \mathrm{~cm}$. 
As curvas ajustadas na figura (2) foram obtidas com a aplicação do método de regressão de terceira ordem por mínimos quadrados e os erros associados ao método (tabela abaixo) foram calculados por chi-quadrado.

\section{Figura 2 - Aplicação do parâmetro de ajuste b ao Benzeno e Tolueno.}
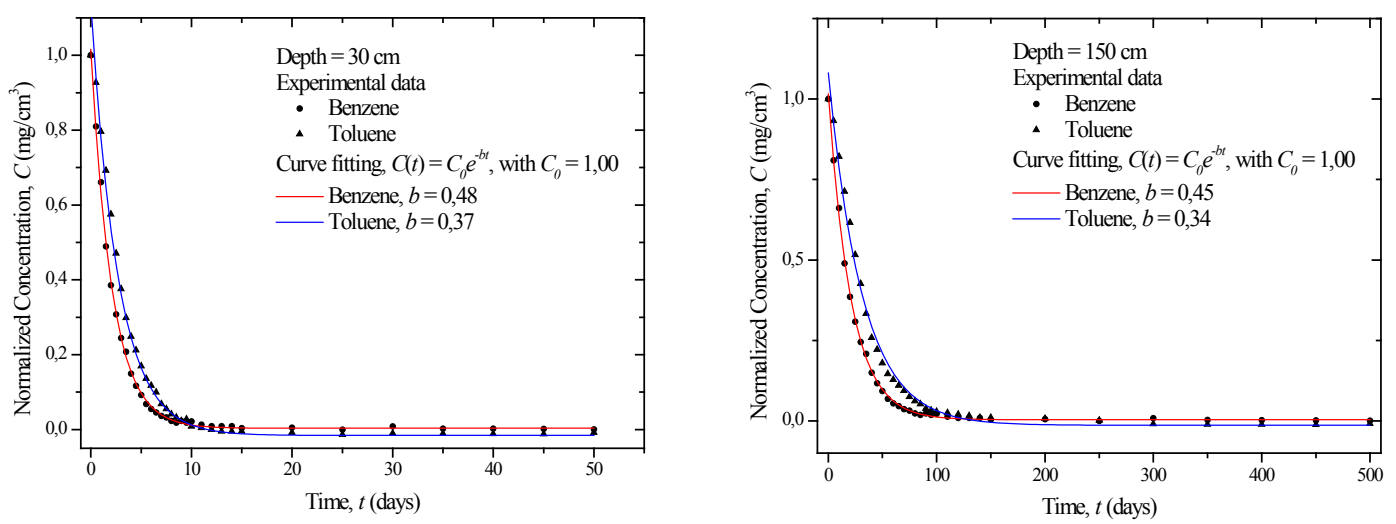

Nos gráficos anteriores, as concentrações nominais foram normalizadas, $C_{0}=1,00 C_{0}=1,00$ , visando homogeneizar esta grandeza em diferentes profundidades e facilitar a comparação dos resultados obtidos. Uma vez normalizados os valores da concentração é possível identificar a simples vista a diferença da figura (1), que a tendência da diminuição da concentração segue a equação (4), no entanto, aparece uma ligeira dependência do valor de b com a profundidade para um mesmo elemento; o que pode ser um indicativo da sua possível relação com o mecanismo físico de lixiviação (MALLANTS et al,2011). Esta variação no parâmetro b, como se desprende dos resultados está dentro da margem de erro das medidas realizadas, logo não altera a forma geral da equação proposta da concentração contra o tempo. O que permite concluir que nossa premissa original continua sendo válida.

Um resultado importante dos dados mostrados na figura (2), é que podemos estabelecer, no caso de derrames de compostos BTEX, o intervalo temporal, limites da integral proposta em (2) em que a concentração de contaminantes no solo, supera os valores estabelecidos pela equação (1) da metodologia geral (CETESB, 2001; CETESB, 2006). Justificando, assim, a inclusão da expressão (2) no valor de referência da dose de ingresso que leva ao resultado mostrado na equação (2).

A utilização da equação (2) para o cálculo da dose de ingresso em acidentes irá propiciar, conhecidos os valores de $b$ para cada elemento contaminante, o estabelecimento de uma previsão mais precisa dos possíveis impactos ambientais e na saúde humana (comunidade / trabalhadores, etc.). Os valores obtidos de $b$, para os compostos BTEX aqui tratados, se mostram na tabela 3 a seguir: 
Tabela 3 - Valores do parâmetro b obtidos para os compostos BTEX.

\begin{tabular}{ccc}
\hline Profundidade $(\mathbf{c m})$ & Composto BTEX & Parâmetro $\boldsymbol{b} \pm \mathbf{0 , 0 8}(\mathbf{1} /$ dias $)$ \\
\hline \multirow{2}{*}{30} & Benzeno & 0,48 \\
& Tolueno & 0,37 \\
& Etilbenzeno & 0,35 \\
& Xilenos & 0,28 \\
\hline \multirow{2}{*}{150} & Benzeno & 0,45 \\
& Tolueno & 0,34 \\
& Etilbenzeno & 0,30 \\
& Xilenos & 0,27 \\
\hline \multirow{2}{*}{640} & Benzeno & 0,44 \\
& Tolueno & 0,36 \\
& Etilbenzeno & 0,33 \\
& Xilenos & 0,27 \\
\hline
\end{tabular}

As equações de transporte (2 e 3), quando aplicadas em uma situação de derrame pontual, apresentarão curvas elevadas, inicialmente no solo, com um rápido crescimento das concentrações lixiviadas e volatilizadas. No solo superficial (camada até $30 \mathrm{~cm}$ ), o soluto impulsionado pelo fluxo superficial das precipitações e também pelo imediato processo de volatilização, levam rapidamente as concentrações dos compostos contaminantes para as faixas mais profundas $(30 \mathrm{a} 150 \mathrm{~cm} / 150$ a $640 \mathrm{~cm}$ ). A figura (1) demonstra claramente essa dinâmica (ŠIMŮNEK e BRADFORD, 2008; SUAREZ, 2007). As concentrações iniciais modeladas se concentram mais na zona superficial do solo, enquanto que, nas zonas mais profundas do solo, ocorre uma atenuação do decaimento das concentrações. De forma geral, verifica-se que o baixo teor de concentração do etilbenzeno e xilenos no combustível diesel, evidenciado na figura 1 , resultarão em probabilidades de risco toxicológico de menor relevância.

Concentrações de exposição nos compartimentos ar, solo residual e água subterrânea.

As concentrações de exposição máximas médias para o benzeno no diesel chegam a 0,0789 $\mathrm{mg} / \mathrm{m}^{3}$ para as concentrações volatilizadas para o ar no ambiente externo (limite orientador $0,0017 \mathrm{mg} / \mathrm{m}^{3}$ ). No solo residual superficial (até $30 \mathrm{~cm}$ ), as concentrações máximas chegam a $1310 \mathrm{mg} / \mathrm{kg}$ (limite de prevenção $0,03 \mathrm{mg} / \mathrm{kg}$ ) e para a água subterrânea chegam a 31,4 mg/1 (valor máximo permitido $0,005 \mathrm{mg} / 1$ ). No caso das concentrações de benzeno na gasolina, os valores são de $0,112 \mathrm{mg} / \mathrm{m}^{3}$ para as concentrações volatilizadas, $1850 \mathrm{mg} / \mathrm{kg}$ para o solo residual superficial e, por fim, 44,5 mg/l para água subterrânea. Todas as concentrações de exposição para gasolina foram ligeiramente superiores ao diesel (CETESB, 2012, FIT benzeno). Destas concentrações de exposição foram estimadas as doses de ingresso diárias médias proporcionais, a variação do valor para o período total de 365 dias ficou na faixa de 0,0001 a 1,65 (mg/kg/1/ $\left.\mathrm{m}^{3}\right)$ para a proporção no diesel e na faixa de 0,0001 a $2,34\left(\mathrm{mg} / \mathrm{kg} / 1 / \mathrm{m}^{3}\right.$.dia) para as proporções de benzeno na gasolina.

Em relação ao tolueno, as concentrações de exposição médias para o diesel no período máximo de simulação (365 dias) foram de $0,0889 \mathrm{mg} / \mathrm{m}^{3}$ (limite orientador $0,26 \mathrm{mg} / \mathrm{m}^{3}$ ) para o tolueno volatilizado para o ar no ambiente externo, $2760 \mathrm{mg} / \mathrm{kg}$ (limite de prevenção $0,14 \mathrm{mg} / \mathrm{kg}$ ) 
para o tolueno no solo residual superficial e 25,9 mg/1 (VMP de 0,024 mg/l) para as concentrações lixiviadas para água subterrânea. Estas concentrações de exposição são médias acumuladas no período total de 365 dias. Para o tolueno presente na gasolina, as concentrações de exposição médias foram de $0,095 \mathrm{mg} / \mathrm{m}^{3}$ para o tolueno volatilizado para o ar no ambiente externo, 2950 $\mathrm{mg} / \mathrm{kg}$ para o tolueno no solo residual superficial e 27,7 mg/l para o tolueno lixiviado para a água subterrânea. (CETESB, 2012, FIT tolueno).

Para o composto etilbenzeno, as concentrações de exposição médias para gasolina, alcançaram no final do período de 365 dias, os níveis de $0,00567 \mathrm{mg} / \mathrm{m}^{3}$ (valor de referência rural $0,002 \mathrm{mg} / \mathrm{m}^{3}$ ) para as concentrações de etilbenzeno volatilizadas para o ambiente externo, nível de $308 \mathrm{mg} / \mathrm{kg}$ (valor de prevenção $6,2 \mathrm{mg} / \mathrm{kg}$ ) para as concentrações residuais no solo superficial e 1,19 mg/1 (VMP 0,2 mg/l) para as concentrações lixiviadas para a água subterrânea (CETESB, 2012, FIT etilbenzeno). Em relação ao etilbenzeno no diesel as concentrações de exposição foram de $0,0152 \mathrm{mg} / \mathrm{m}^{3}, 823 \mathrm{mg} / \mathrm{kg}$ e $3,19 \mathrm{mg} / 1$ respectivamente.

Por fim, para os xilenos, as concentrações de exposição médias acumuladas foram maiores no diesel, com $0,0199 \mathrm{mg} / \mathrm{m}^{3}$ (limite de referência varia entre 0,003 a $0,39 \mathrm{mg} / \mathrm{m}^{3}$ ) para as concentrações volatilizadas, $1120 \mathrm{mg} / \mathrm{kg}$ (valor de prevenção $0,13 \mathrm{mg} / \mathrm{kg}$ ) para as concentrações no solo superficial residual e $6,35 \mathrm{mg} / 1$ (VMP 0,3 mg/l) para as concentrações lixiviadas para água subterrânea (concentrações médias após 365 dias de exposição). Para a gasolina as concentrações respectivas foram, 0,00319 mg/m³, $179 \mathrm{mg} / \mathrm{kg}$ e 1,02 mg/1 (CETESB, 2012, FIT xilenos).

Risco toxicológico individual por via de exposição.

A partir dos cenários conceituais modelados, as vias de exposição adotadas foram: Ingestão oral de água subterrânea ou superficial; Contato dérmico com água subterrânea ou superficial; Ingestão oral de solo superficial; Contato dérmico com solo superficial; Ingestão oral de água durante recreação/natação e Inalação de vapores no ambiente externo. As doses de ingresso estimadas para cada via de exposição foram estimadas considerando a evolução e o decaimento gradual das concentrações nos compartimentos (ar, solo residual e água). Para o benzeno, após estimativa das doses de ingresso diárias para cada via de exposição, verificou-se que para ambos os casos (diesel e gasolina) a via de exposição mais relevante quanto ao risco foi à ingestão oral de solo superficial, chegando à probabilidade de risco carcinogênico de 0,0513 para o benzeno no diesel e de 0,0727 para o benzeno na gasolina. Salienta-se que conforme a CETESB (2006) o nível aceitável baseado no risco (NABR) para compostos carcinogênicos é de 0,00001. 
Figura 3 - Risco toxicológico por via de exposição para o benzeno (Cs - solo, Caar e Cw - água).

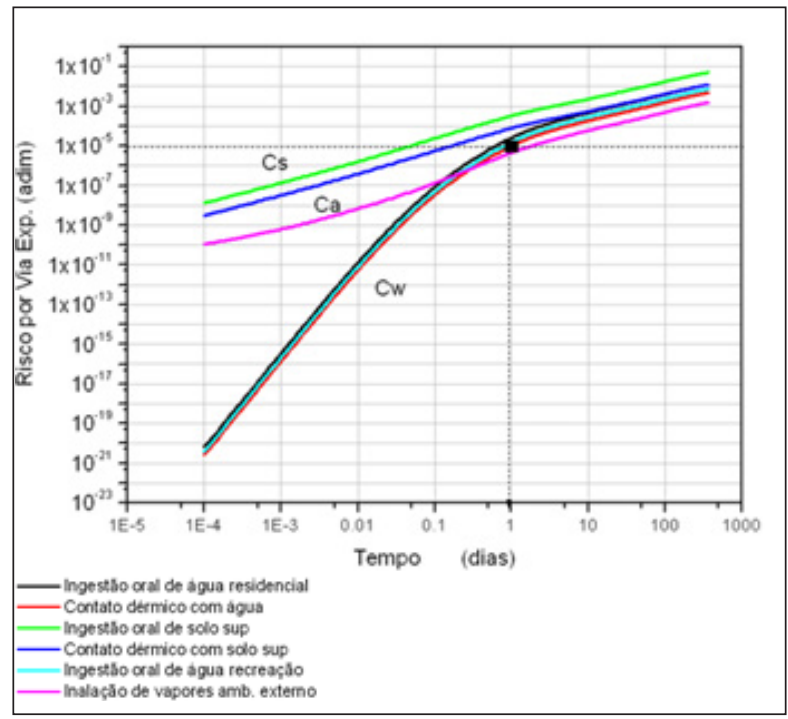

Observa-se em relação ao tolueno, que as doses de ingresso estimadas no período final de 365 dias para as vias relacionadas à ingestão oral do contaminante foram de $2,42 \mathrm{mg} / \mathrm{kg} / \mathrm{l}$.dia, para o contato dérmico foi de $0,561 \mathrm{mg} / \mathrm{kg} / 1$ dia e para a inalação de vapores de $0,0672 \mathrm{mg} / \mathrm{m}^{3}$, os respectivos valores de referência conforme a tabela (2) são 0,2 (oral) 0,16 (dermal) e 0,114 (inalatória) conforme IRIS U.S. EPA (2004). Para os casos de ingestão oral e contato dérmico com o solo ou água, as doses de ingresso individuais diárias estão muito acima das doses de referência, com isso, a predição da ocorrência de efeitos toxicológicos é dado pelo quociente de risco, que tem como taxa limite 1 . Na ocorrência de uma relação RfD e Dose de Ingresso superior a 1, haverá a ocorrência de efeitos adversos toxicológicos, que pode variar de simples náuseas a efeitos letais. Até 10 dias após o acidente de contaminação do solo com tolueno, o quociente de risco fica abaixo da taxa limite 1, após esse período existe uma elevação que pode chegar a 16,2 de risco considerando o tolueno em gasolina (figura 4).

Figura 4 - Risco toxicológico por via de exposição para o tolueno (Cs - solo, Caar e Cw - água).

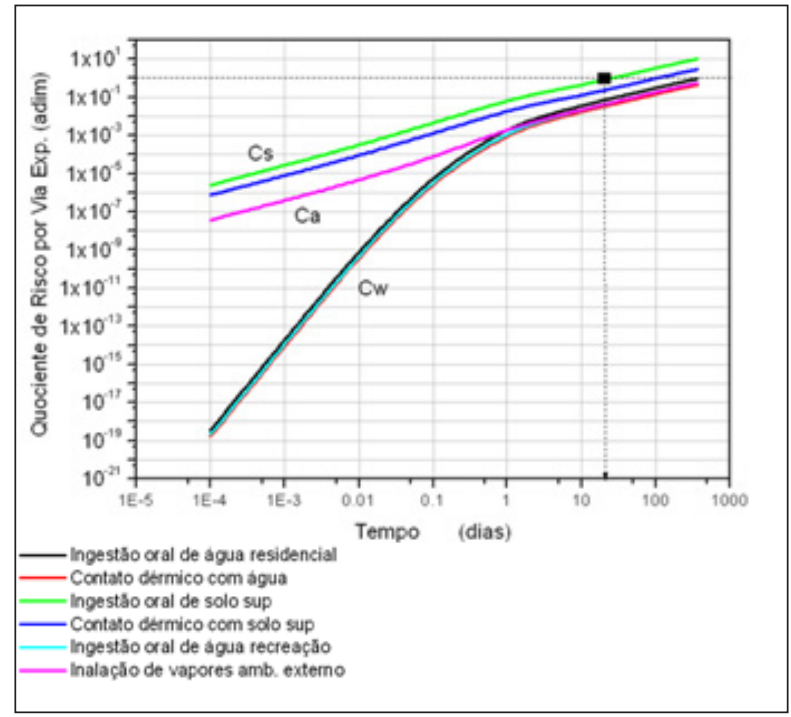


Para o etilbenzeno, o risco por via de exposição ultrapassa o limite de risco de 0,00001 antes do período de 1 dia (para a via de ingestão oral do solo superficial). O etilbenzeno no diesel alcança a probabilidade de risco máximo para as vias de ingestão oral de 0,0024 e para o contato dérmico, a probabilidade máxima é de 0,000444; para a inalação de vapores de 0,0000413. $\mathrm{O}$ etilbenzeno na gasolina alcança o risco máximo para ingestão oral de 0,000898 , para o contato dérmico foi de 0,000166 e para a inalação de vapores de 0,0000154. Todas as vias apresentaram risco mais elevado para o diesel do que para a gasolina.

As probabilidades de risco carcinogênico para o etilbenzeno foram menores do que o benzeno em magnitude. No que se refere a sua elevação com o tempo, há uma clara diferença entre a simulação com o diesel e com a gasolina. Adotando que o acidente tenha ocorrido para 1000 litros de diesel, o etilbenzeno deveria ser remediado antes de $24 \mathrm{~h}$ para que não pudesse ultrapassar o limite de risco estabelecido, que, neste caso, alcança 0,0000192. Adotando o acidente com 1000 litros de gasolina, o etilbenzeno alcança a probabilidade de risco limite a partir do $3^{\circ}$ dia, com 0,0000185 .

\section{Figura 5 - Risco toxicológico por via de exposição para o etilbenzeno (Cs - solo, Ca- ar e Cw - água).}

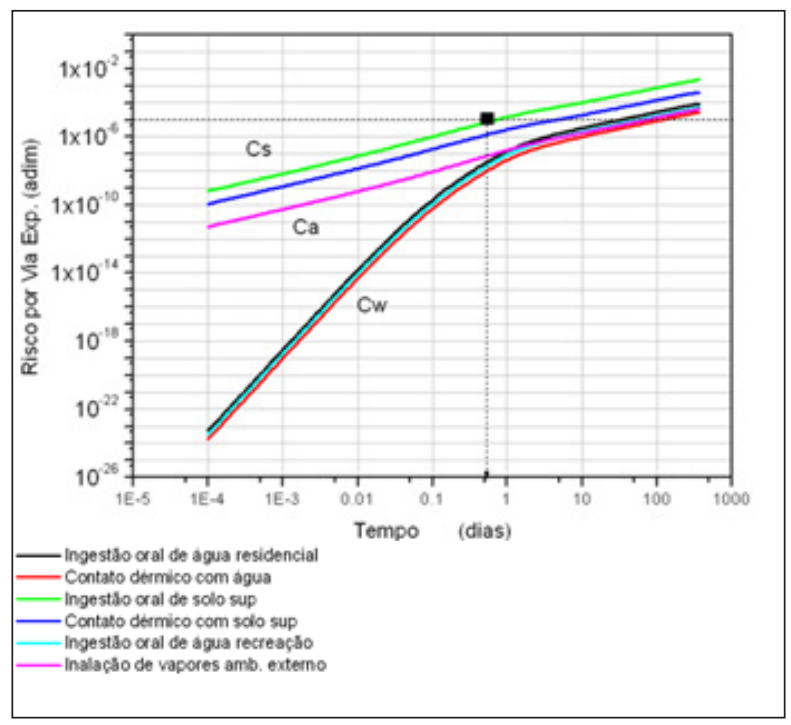

A estimativa do risco não carcinogênico para os xilenos apresentou, para o diesel, maior quociente de risco, algo semelhante com o etilbenzeno; os xilenos, na gasolina, ficaram abaixo do índice de risco limite. A via de maior relevância foi à ingestão oral de solo superficial, assim como em todos os casos apresentados, com um quociente de 3,99 para o diesel (acima do HQ) e de 0,638 para a gasolina (neste caso, abaixo do HQ aceitável).

A única via de exposição que ultrapassa o limite do quociente de periculosidade (HQ) é a ingestão oral de solo contaminado superficial (diesel), o que acontece após 100 dias de exposição acumulada por esta via. O risco não carcinogênico total, torna-se preocupante e sujeito a processos de intervenção após 50 dias de exposição acumulada aos xilenos, caso o acidente fosse com o diesel. Adotando a gasolina como combustível contaminante o risco não carcinogênico total fica abaixo do limite $H$, durante todo o período de simulação, porém analisando qualitativamente a tendência das curvas, o risco não carcinogênico pode ser superado após 365 dias de contaminação, conforme figura (6). 


\section{Figura 6 - Risco toxicológico por via de exposição para o xilenos (Cs - solo, Ca- ar e Cw - água).}

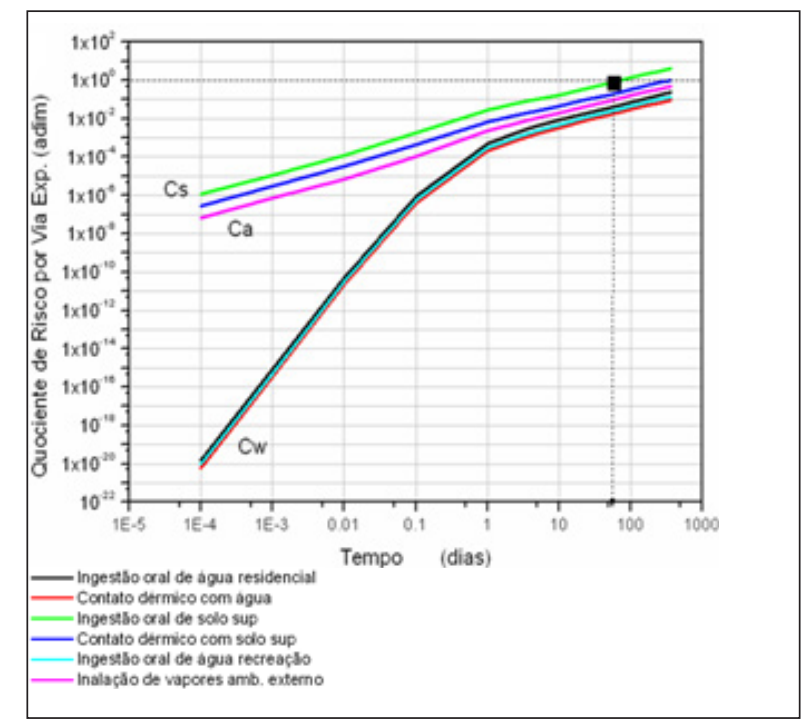

\section{Risco toxicológico dinâmico total}

Após somatória do risco individual por via de exposição, os gráficos da figura 7 mostram o risco total conforme equação (6 e 7) para o BTEX proporcional ao diesel e gasolina. O risco total final estimado para o benzeno no diesel foi de 0,08962 e para a gasolina foi de 0,1271, sabese que conforme metodologia ACBR o nível de risco aceitável total é de 0,00001 (CETESB, 2006). Probabilidades que quando aplicadas a uma população de 1710 pessoas representam $9 \%$ e $13 \%$ da população respectivamente, isto é, algo entre 153 a 222 pessoas com possibilidade de desenvolver câncer durante seu tempo de vida, considerando uma exposição aguda ao benzeno derivada de um acidente com infiltração pontual do volume de 1000 litros de diesel ou gasolina. Observa-se que o limite de risco é superado em menos de $24 \mathrm{~h}$, depois de ocorrido o acidente ambiental com diesel ou gasolina na jazida de areia silicosa. Desta forma, a tomada de decisão para casos de contaminação como esses, devem ser tomadas rapidamente, visando à aplicação de processos de remediação imediatos. As probabilidades de risco variam de 0,00000001 (risco mínimo) e chegam a 0,1 (risco crítico para o período).

Para o tolueno é apresentado o cálculo do risco toxicológico não carcinogênico de forma dinâmica, com variações mínimas e máximas do quociente de risco $(H$ ) de 0,00000336 e 16,2 (para gasolina). Em até 10 dias após o acidente de contaminação do solo, o quociente de risco fica abaixo da taxa limite referencial 1 (CETESB, 2006). Após esse período existe uma elevação que ultrapassa o limite mínimo estabelecido. É preciso compreender, no entanto, que a avaliação do risco não carcinogênico, necessita de uma análise mais detalhada, verificando o potencial de cada quociente de risco mensurado e seus efeitos reais sobre a saúde da população.

As probabilidades máximas de risco carcinogênico alcançadas com o etilbenzeno foram de 0,00108 para a gasolina e de 0,00289 para o diesel, ou seja, uma variação percentual de 0,11\% a 0,29\%, o que representa para a população em estudo (1710 habitantes) o quantitativo de 2 a 5 pessoas com possibilidade de desenvolver câncer devido à exposição ao contaminante etilbenzeno. 
Analisando a exposição conjunta do etilbenzeno com o benzeno, bastaria somar as probabilidades estimadas, elevando o quantitativo total.

Para os xilenos, o risco não carcinogênico total $(H)$, torna-se preocupante e sujeito a processos de intervenção após 50 dias de exposição acumulada, caso o acidente fosse com o diesel. Adotando a gasolina como combustível contaminante, o risco não carcinogênico fica abaixo do limite estabelecido pela metodologia ACBR da CETESB (2006) que é igual a 1 (durante todo o período de simulação). Assim, o risco total não carcinogênico alcança o quociente máximo de 5,94 para o diesel, sendo que a maior parte do período de exposição, os índices ficam abaixo do limite referencial estabelecido $(H<1)$. No caso de acidente com a gasolina o risco total máximo alcançado para o período simulado foi de 0,951 , índice que pode ser superado após 1 ano de exposição.

\section{Figura 7 - Risco toxicológico total para os compostos BTEX}
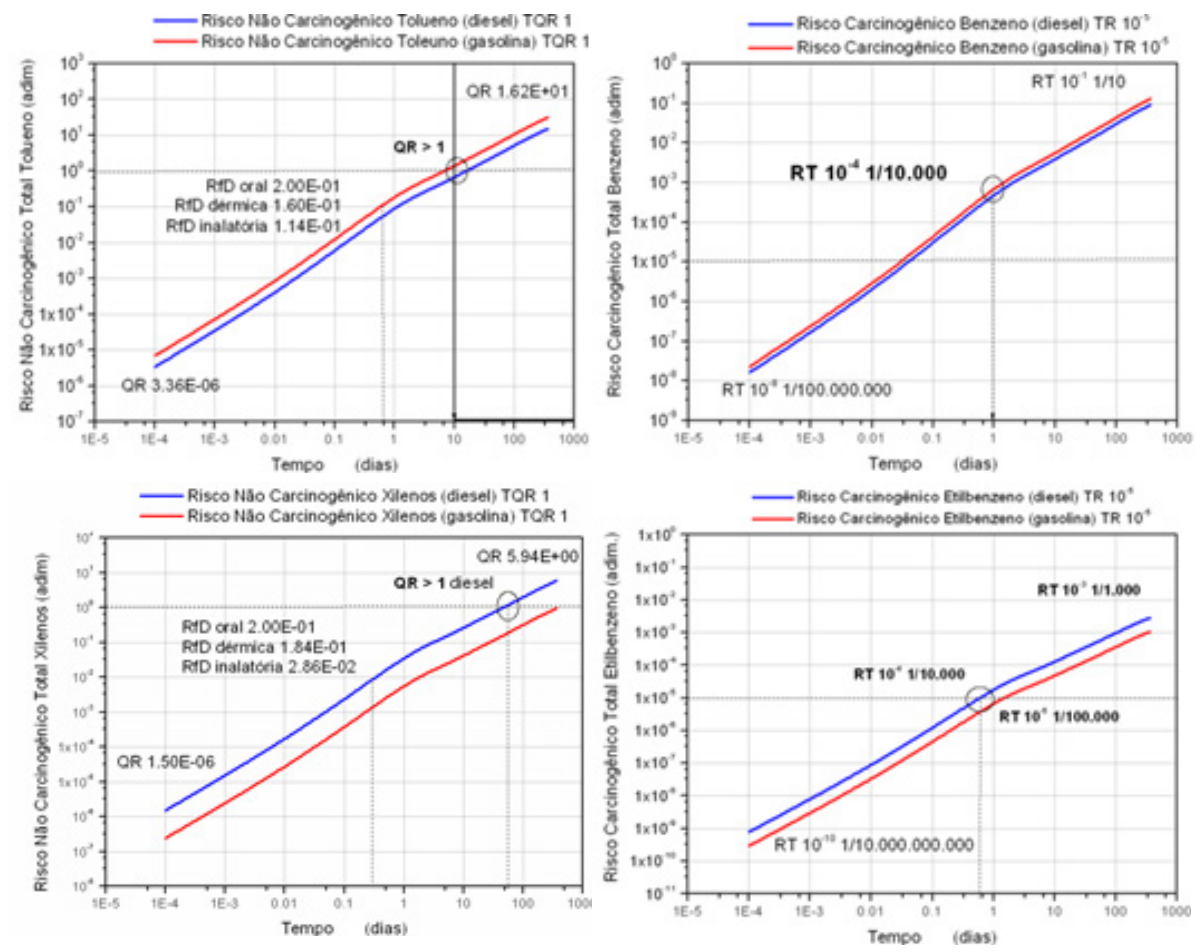

${ }^{*} \mathrm{TQR}=\mathrm{HI}$ quociente de risco $/ \mathrm{TR}=$ Risco Total

\section{Conclusões}

Pode-se afirmar que há evidentes possibilidades de ocorrência de riscos toxicológicos carcinogênicos e não carcinogênicos reais para a população. O limite do risco toxicológico $(0,00001)$ é suplantado em apenas $2,4 \mathrm{~h}$ para o benzeno e em $16,8 \mathrm{~h}$ para o etilbenzeno, tomando como referência o limite NABR da CETESB (2006), com vias de maior risco vinculadas a ingestão oral e contato dérmico com a água e solo contaminados. Para os compostos não carcinogênicos, o quociente de periculosidade padrão, denominado HQ, de valor 1, é ultrapassado em 10 dias para o tolueno e em 50 dias para o xilenos. 
A simulação realizada estimou probabilidades de risco toxicológico carcinogênicos, considerando o diesel e a gasolina, com valores mínimos em 0,00000001 e máximos 0,1 para o benzeno. Para o etilbenzeno essa variação foi de 0,0000000001 (valor mínimo) e 0,001 (valor máximo). Para os compostos não carcinogênicos, a variação do quociente de risco total ( $H$ ) foi de 0,000001 a 16 para o tolueno e de 0,000001 a 6 para o xilenos.

A avaliação do risco toxicológico à saúde humana, considerando aspectos integrados de modelagem matemática e simulação numérica através do Hydrus $1 D$, com aplicação da metodologia $A C B R$ da CETESB (2001) e CETESB (2006) e, empregos do modelo de avaliação da exposição, aqui denominados, Dinâmico Proporcional, permitiram estimar as concentrações de exposição e a probabilidade de ocorrência de efeitos adversos a saúde humana por BTEX. A adaptação efetuada na metodologia $A C B R$ utilizada pela CETESB (2006), permitiu a análise do risco de forma dinâmica, sendo possível estimar a evolução gradual do risco, traçando de forma objetiva sua tendência de crescimento.

A utilização do Hydrus 1D, na modelagem de contaminação de solo por BTEX, foi eficiente na mensuração unidimensional das concentrações. Foi necessário definir as propriedades físicas e hidráulicas específicas do solo, como também adaptar e aplicar outras formulações para quantificar as concentrações finais de exposição, ou seja, concentrações iniciais obtidas pelo modelo Hydrus $1 D$ foram recalculadas considerando os processos de atenuação natural de volatilização e lixiviação. Os fatores de atenuação natural, como o fator de volatilização e lixiviação, conforme estabelece a metodologia da CETESB (2006), possibilitaram a estimação mais precisa das concentrações reais de exposição. O código computacional permitiu também, prever as concentrações iniciais médias em dimensões singulares (solo e/ou água), sendo que as concentrações de transferência entre as fases foram obtidas por meio de cálculos utilizando as equações de atenuação natural (NAF), principalmente, com relação à fase vapor e à fase lixiviada dos contaminantes (MAZZUCO, 2004).

A utilização do Hydrus $1 D$ possibilitou a realização de cálculos de concentrações realísticos, validados na literatura específica, considerando os efeitos cíclicos e variáveis da atmosfera, como também a complexidade estrutural (física e química) dos compostos BTEX e do ambiente subterrâneo como um todo. Apesar de seu processo de simulação ser apenas unidimensional, isto é, fluxo verticalizado de concentrações, sua eficiência foi confirmada na aplicação em solos arenosos de média porosidade.

O presente estudo caracterizou-se por utilizar modelos numéricos para a compreensão do processo de infiltração e estimação das concentrações em diferentes compartimentos do solo de forma preditiva e dinâmica, subsidiando a avaliação do risco. Salienta-se que, usualmente, os processos de avaliação de risco são aplicados em áreas já contaminadas, casos em que há uma intensa necessidade de dados reais de monitoramento em campo. Neste estudo, o enfoque foi à adaptação e melhoria da metodologia de quantificação do risco toxicológico $R B C A$ da ASTM (2000), utilizada no Brasil pela CETESB (2001) e CETESB (2006), com ênfase na prevenção, sendo aplicável em possíveis áreas com potencial de poluição por BTEX. Resulta assim, em um método que possibilita a observação de informações e a tomada de decisões para minimização dos efeitos adversos a saúde humana, de forma preventiva, sendo aplicável a qualquer tipo de contaminante orgânico em diferentes estruturas de solo. 


\section{Agradecimentos}

Os autores agradecem a instituições CAPES / CNPQ, pelos recursos aplicados no desenvolvimento da pesquisa.

Referências

ASTM. Standard Guide for Risk-Based Corrective Action: American Society for Testing and Materials - E 2081- 00.2000.

CETESB, Evaluation of toxicological risk to human health 9000, Cetesb GTZ Project Chapter IX, São Paulo, 2001.

CETESB, Risk-based corrective actions (ACBR) applied to areas contaminated with petroleum hydrocarbons and other liquid fuels. Annex VII, São Paulo, 2006.

CETESB, Sheet toxicological: Benzene, Cetesb, Division of Toxicology, Genotoxicity and Environmental Microbiology, São Paulo, 2012.

MIHELCIC, J. R. e ZIMMERMAN, J. B. Engenharia Ambiental: fundamentos, sustentabilidade e projeto. LTC, Rio de Janeiro, 2012.

COELHO, Mário José. Industrial Sand, MME-SGE, 44 Sand Industrial Product, Technical Report 44 Technical Assistance Project to Energy Sector, Brazil, 2010.

CORDAZZO, Jonas. Modeling and numerical simulation of pouring gasoline plus ethanol in groundwater. Federal University of Santa Catarina, the Graduate Program in Mechanical Engineering, Florianopolis, 2000.

EMBRAPA, National Research Center of Soil. Manual methods of soil analysis. CNPS, 2nd ed. rev. current. Rio de Janeiro. 1997.

HURST, Marc V. Hydrologic effects of sand mining in Lake County, Florida. Geological Independent Services, Inc., ER Jahna Industries, Inc. Davenport, FL, 2002.

JURY, and WA HORTON, Robert. Soil physics, 6th ed, edit.Wiley, 2004.

KUYUCAK, Nural. Selecting suitable mining methods for treating effluents. Golder Associates Ltda., Ontario, Canada, 2006.

LEIJ, Feike J. et al. Hydraulic properties of soils Subjected to aqueous solutions with diesel or ethanol-blended diesel, Elsevier, Geoderma 162, pg.288-295, 2011.

MALLANTS, Dirk; TH. VAN GENUCHTEN, Martinus; ŠIMŮNEK, J.; JACQUES, Diederik AND SEETHARAM, SURESH. Leaching of contaminants to groundwater, Swartjes FA (ed.), Dealing with Contaminated Sites, Springs Science, p. 787-850, 2011. 
MAZZUCO, Lilian Maria. Natural attenuation of aromatic hydrocarbons in aquifers contaminated with diesel oil. Master Thesis, Department of Chemistry, Graduate Diploma in Analytical Chemistry, UFSC, 2004.

MIHELCIC, James R. and ZIMMERMAN, Julie Beth. Environmental Engineering: Fundamentals, design and sustainability. LTC, Rio de Janeiro, 2012.

MINISTRY OF MINES AND ENERGY (MME). Industrial Sand. Technical Report 44, Department of Geology, Mining and Mineral Processing - SGM, Consultant Jose Mario Coelho, J. Mendo Consulting, Brasilia, 2010.

MOREIRA, Mark Donadello. High purity silica sand Santa Maria Eternal: Belmonte, Bahia. Salvador, CBPM, 2005.

MUALEM Y. A new model for predicting the hydraulic conductivity of unsaturated porous media Water Resour. Res.; 12 (3) 513-522, 1976.

OLIVEIRA, Ricardo Nunes de Castro; CAMPOS, Rosiany Possati; HADDAD, Assed Naked. Risk analysis of the sand extraction sector, XIX BRAZILIAN SYMPOSIUM ON WATER RESOURCES, Brazilian Association of Water Resources, 2011.

PIGGOTT, John H.; CAWLFIELD, Jeffrey D. Probabilistic sensitivity analysis for one-dimensional contaminant transport in the vadose zone, Journal of Contaminant Hydrology, n.24, Elsevier Science, 1996.

RUBIO, Carles.Hydrodynamics of them suelos an area montaña average Mediterranean sometida the exchange of use y cubierta. Universitat Autònoma de Barcelona, Consejo Superior de Investigaciones Científicas, Institut Ciències La Terra “Jaume Almera”, 2005.

ŠIMŮNEK, J and BRADFORD, Scott. Vadose zone modeling: Introduce on and Importance, Vadose Zone Journal, vol. 7, n.2, Soil Science Society of America, 2008.

ŠIMŮNEK, J., M. SEJNA, M. SAKAI, H. SAITO, M. and TH VAN GENUCHTEN, The HYDRUS-1D software package for simulating the one-dimensional movement of water, heat, and multiple solutes in variably-saturated media Version 4.0x, Hydrus Series 3, Department of Environmental Sciences, University of California Riverside, Riverside, CA, USA, 2008.

SUAREZ, Francisco et al. Transport of simazine in unsaturated sandy soil and predictions of its leaching under hypothetical field conditions, Elsevier, Journal of Contaminant Hydrology 94, pg.166-177, 2007. 
U.S. ENVIRONMENTAL PROTECTION AGENCY (U.S.EPA). Using Soil Guideline Values. Science Report SC050021/SGV introduction. Bristol: Environment Agency. Soil Guideline Values for benzene in soil Science Report SC050021 / benzene SGV, 2009.

U.S. GEOLOGICAL SURVEY (USGS). Minerals yearbook 2011: silica advance release, U.S. Department of the Interior, Washington, DC, USA, March, 2013.

WISCONSIN GEOLOGICAL SURVEY (WGS). Silica sand mining in Wisconsin. Wisconsin Department of Natural Resources, Madison, WI, 2012.

XU, M. and ECKSTEIN, Y. Use of Weighted Least-Squares Method in Evaluation of the Relationship Between dispersity and Field Scale, Ground Water, Vol 33, n. 6, p.905-908, 1995.

PNUMA, IPCS, Evaluación de riesgos químicos: evaluación de riesgos humanos, evaluación de riesgos ambientales evaluación de riesgos ecológicos Módulo de capacitación no. 3, PNUMA/IPCS, 1999.

PENNER, G. C. Estudos laboratorias da contaminação do solo por gasolina com o uso de detetor de fotoionização. Dissertação de Mestrado, Escola de Engenharia de São Carlos, USP, 2000.

CETESB, Ficha de informação toxicológica: Benzeno. Divisão de Toxicologia, Genotoxicidade e Microbiologia Ambiental. São Paulo, 2012.

CETESB, Ficha de informação toxicológica: Etilbenzeno. Divisão de Toxicologia, Genotoxicidade e Microbiologia Ambiental. São Paulo, 2012.

CETESB, Ficha de informação toxicológica: Tolueno. Divisão de Toxicologia, Genotoxicidade e Microbiologia Ambiental. São Paulo, 2012.

CETESB, Ficha de informação toxicológica: Xilenos. Divisão de Toxicologia, Genotoxicidade e Microbiologia Ambiental. São Paulo, 2012.

DONAIRE, P. P. R. Tratamento de água subterrânea contaminada com BTEX utilizando fotocatálise heterogênea. Tese de Doutorado, Programa de Pós-Graduação em Química, Universidade Estadual de Campinas, 2007.

FINOTTI, A. R. CAICEDO, N. O. L., RODRIGUES, M. T. R. Contaminação subterrâneas com combustíveis derivados de petróleo: Toxicidade e a legislação brasileira. Ver. Brás. de Recursos Hídricos - RBRH, V. 6 n. 2, 2001.

JURY, W.A.; HORTON, R. Soil physics, 6th ed, edit. John Wiley \& Sons Inc. 2004 
KLUTE,A.; DIRKSEN, C. Conductivities and diffusivities of unsaturated soils. Methods of soil analysis. Part, v. 1, p. 687-734, 1986.

MAZZUCO, L. M. Atenuação natural de hidrocarbonetos aromáticos em aqüíferos contaminados com óleo diesel. Dissertação Mestrado, Departamento de Química, PósGraduação em Química Analítica, UFSC, 2004.

TODD, D. K.; MAYS, L. W. Groundwater hydrology, Third Edition, John Wiley \& Sons, Inc. Hoboken, USA, 2005.

VAN GENUCHTEN, M. TH. A closed form equation for predicting the hydraulic conductivity of unsaturated soils, Soil Sci. Soc. Am. J., 44, 892 898, 1980a.

VAN GENUCHTEN, M. TH. Determining transport parameters from solute displacement experiments. Report N.118, U. S. Salinity Laboratory, USDA, ARS, Riverside, CA, 1980b. 